\title{
Van Wyk- Grumbach Syndrome - An Unusual Presentation of Severe Hypothyroidism
}

\author{
Naznin $L^{1}$, Saha $S^{2}$, Saha $D^{3}$, Sultana $S^{4}$, Chowdhury $M J^{5}$
}

\begin{abstract}
Van Wyk- Grumbach syndrome (VWGS) is characterized by juvenile hypothyroidism, delayed bone age, and precocious puberty with a complete reversal to the pre-pubertal state following thyroid hormone replacement therapy. In this study, a 7 years and 1 month old girl presented with precocity having premature menarche, short stature, constipation, delayed bone age and enlarged bilateral multicystic ovaries. She presented with acute abdomen due to torsion of left ovary and had to undergo left sided oophorectomy and right ovarian cystectomy. High serum $\mathrm{TSH}$, low $\mathrm{FT}_{4}$ with high FSH but low LH within pre-pubertal range suggestive of 'severe hypothyroidism with $\mathrm{FSH}$ dominant precocious pseudopuberty' confirmed the diagnosis of VWGS.
\end{abstract}

Key-words: Grumbach syndrome, hypothyroidism, precocious pseudopuberty.

\section{Introduction}

Kendle, in 1905, first described the association of severe primary hypothyroidism and isosexual precocious puberty in females ${ }^{1,2}$. In 1960, Van Wyk and Grumbach first reported the clinical syndrome of long standing primary hypothyroidism associated with isosexual precocious pseudopuberty and multicystic enlarged ovaries in young female child ${ }^{1-5}$. Boys of VWGS having macroorchidism with no significant signs of virilization have also been reported $^{1,2}$. In this study, a 7 year and 1 month old girl is presented with bilateral enlarged ovaries with twisted left ovarian cyst, isosexual precocious puberty, short stature and constipation due to unnoticed and untreated long standing hypothyroidism.

\section{Case Report}

A girl of 7 years 1 month reported to emergency department of a private hospital with the features of acute abdomen having left sided abdominal pain and vomiting for several hours. She also had history of scanty per vaginal bleeding for the preceding 7 days, but no history of fever or burning micturition. She was of short stature in respect of her age; often suffered from constipation since her 3 years of age. Her parents mentioned that she was less sweaty even in summer and was slow in every aspect since her 3 years of age. The girl was a meritorious student of her class but her performance was gradually deteriorating. She was born of non-consanguineous parents at full-term through lower uterine caeserian section (LUCS) and the milestone of development was normal. General examination revealed, she was restless due to pain in abdomen, her pulse rate was $124 /$ min (though pulse rate recorded on previous occasion was $64 / \mathrm{min}$ ), temperature $98^{\circ} \mathrm{F}$ and was mildly dehydrated. Her height was $104 \mathrm{~cm}(<3 \mathrm{rd}$ centile) ( $z$ score -3.77), weight was $21 \mathrm{~kg}$ (26th centile) ( $z$ score -0.5 ), in contrast 50 th centile weight for age $22 \mathrm{~kg}$ and height for age $121 \mathrm{~cm}$. Her thyroid gland was not enlarged. As per Tanner's staging, her sexual maturation score was $\mathrm{B} 1$ and $\mathrm{PH} 1$ for breast and pubic hair, respectively, and her axillary hair was absent. On palpation, abdomen was soft, non-distended, but tender on right and left iliac fossa. She had mild anaemia with haemoglobin level of 9.5 $\mathrm{gm} / \mathrm{dl}$, ESR $15 \mathrm{~mm}$ in 1st hour and non specific findings in peripheral blood film. Urine routine examination revealed no abnormality. Fasting blood sugar $5.1 \mathrm{mmol} / \mathrm{L}$. Hormonal investigations revealed $\mathrm{TSH}>100 \mu \mathrm{lU} / \mathrm{ml}$ (normal range $=0.7-5.7$ ), free $\mathrm{T}_{4}$ $0.9 \mathrm{pmol} / \mathrm{L}$ (normal range $=11.8-24.6$ ), total $\mathrm{T}_{4} 13.6$

1. Lt Col Lubna Naznin, MBBS, MCPS, DCP, FCPS(Biochemistry), Classified Spl in Pathology, AFIP, Dhaka; 2. Dr Suchitra Saha, MBBS, DGO, FCPS(Gyne \& Obs), Gynaecologist, Mohammadpur Fertility Service \& Training Centre (MFST), Mohammadpur, Dhaka; 3. Maj Gen Debashish Saha, MBBS, FCPS(Biochemistry) MMed, Commandant, AFIP, Dhaka; 4. Lt Col Sarmin Sultana, MBBS, MCPS(Clinical Path), Graded Spl in Pathology AFIP, Dhaka; 5. Dr Md Jahangir Chowdhury, MBBS, FCPS(Paed), MD(Paed), Associate Professor of Paediatric, Institute of Child \& Maternal Health (ICMH), Matuail, Dhaka. 
$\mathrm{nmol} / \mathrm{L}$ (normal range $=66-181$ ), suggestive of severe hypothyroidism and anti-TPO was positive with 888 $\mathrm{IU} / \mathrm{ml}(<5.61)$ confirmed autoimmune thyroiditis, $\mathrm{FSH} 8.0 \mathrm{mIU} / \mathrm{ml}$ (normal range=0.2-5.8), $\mathrm{LH}<0.08$ $\mathrm{mIU} / \mathrm{ml}$ (normal range $=0.7-2.0$ ), suggestive of FSH dominant precocious pseudopuberty, Basal Growth Hormone $2.0 \mathrm{ng} / \mathrm{ml}$ (normal range=0.06-5.0), Morning Serum Cortisol $304 \mathrm{nmol} / \mathrm{L}$ (normal range $=138-690$ ). Her radiological bone age was about $3 \frac{1}{2}$ years. USG revealed grossly enlarged ovaries, left ovary $148 \mathrm{~cm}^{3}$ and right ovary $86 \mathrm{~cm}^{3}$ with bilateral large ovarian cysts having multiple internal septations. Left ovarian cyst measuring about $75.7 \times 65.4 \times 66.5 \mathrm{~mm}$ and right ovarian cyst measuring about $57.8 \times 45.2 \times 63.2 \mathrm{~mm}$, and uterus measuring $6.1 \times 1.7 \mathrm{~cm}$. Possibility of torsion of left ovary could not be ruled out by USG. After clinical evaluation and supportive investigations, she was diagnosed as a case of 'torsion of left ovary with right ovarian cyst with severe hypothyroidism'. Exploratory laparotomy was done and after opening of the peritoneum left ovarian cyst was found gangrenous, so left oophorectomy was done. Right ovary was healthy, so right ovarian cystectomy was carried out. Histopathology revealed follicular cyst with haemorrhage within the stroma of left ovary and follicular cyst of right ovary. Replacement therapy with tab. thyroxine was initiated and carefully dose was adjusted. So, this young female child is a classic presentation of the Van Wyk-Grumbach syndrome consisting of primary hypothyroidism, isosexual precocious pseudopuberty and multicystic enlarged ovaries. She suffered more complications due to unnoticed and untreated long standing primary hypothyroidism. On follow up she had no further episodes of vaginal bleeding and all her hormones returned to normal. At her last review, after 6 months of thyroid hormone replacement therapy, her height was $113 \mathrm{~cm}$ (<3rd centile) and weight was $19 \mathrm{~kg}$ (3rd centile), meaning that she had lost her weight than before and attained a height of $9 \mathrm{~cm}$ by 6 months with thyroid hormone therapy.

\section{Discussion}

Van Wyk - Grumbach syndrome (VWGS) is a rare disorder. Incidence of VWGS is not known. Only sporadic cases are reported. Clinically VWGS is often a diagnostic challenge, because long-standing primary hypothyroidism in children is known to cause delayed puberty as well as growth delay, whereas, in this rare syndrome, hypothyroidism leads to growth delay with paradoxical precocious puberty ${ }^{5,6}$.

In 1905, Kendle first reported an "astonishing case" of a 9-year-old girl, who had menarche at age 5, fully developed breasts, and the clinical symptoms of female cretinism. After treatment with thyroid extract, her growth resumed, her menstruation stopped, and her symptoms of cretinism resolved ${ }^{6}$. Girls with VWGS have breast development with or without galactorrhoea, follicular cysts of ovaries causing bilateral ovarian masses, and menstruation in absence of pubic or axillary hair, whereas, boys have macroorchidism i.e. testicular enlargement with minimal penile enlargement but no significant signs of virilization $^{1-6}$. Sexual precocity in VWGS is always isosexual and incomplete with lack of pubic and axillary hair growth. Delayed bone age, and high TSH with low $\mathrm{T}_{4}$ are characteristic of $\mathrm{VWGS}^{1,2}$.

In contrast, other causes of precocity are usually associated with increase in linear height, advanced bone age and epiphyseal fusion leading to final short stature ${ }^{2}$. For this, delayed bone age in patients with precocious puberty appears to be an important clue for the diagnosis of VWGS ${ }^{1}$. Usual consistent finding of VWGS is autoimmune thyroiditis and peri-pubertal onset of symptoms. FSH dominated pre-pubertal response with suppressed LH confirms $\mathrm{GnRH}$-independent precocious pseudopuberty in VWGS $^{3}$. The secretion of gonadotropin or gonadal steroids independent of pulsatile GnRH stimulation leads to $\mathrm{GnRH}$-independent sexual precocity. Pituitary enlargement may occur due to thyrotroph hyperplasia in VWGS $^{1}$ which may lead to misdiagnosis of pituitary tumour and may cause expansion of sella turcica ${ }^{5}$. So, it is important to recognize this syndrome because initiating simple thyroid hormone replacement completely resolves the symptoms and hormone abnormalities, avoiding unnecessary investigations for malignancies or surgical intervention ${ }^{2}$. Similar to other reported cases this patient also exhibited breast development vaginal bleeding, and bilateral ovarian masses due to multicystic ovaries. The exact mechanism of development of precocious puberty in VWGS still remains speculative. Van Wyk and Grumbach postulated that an "overlap" in negative feedback response or non-specificity of stimulated by $\mathrm{TRH}$, due to low thyroid hormones ${ }^{1,3,4}$. Alternatively, others 
have suggested that the proximate nature of the TRH centre to the $\mathrm{GnRH}$ centre in the hypothalamus leads to excessive production of both the releasing factors ${ }^{4}$. Another explanation is, pituitary gonadotropins(FSH, LH) are glycoprotein hormones like $\mathrm{TSH}$ and all have a common alpha subunit, can be triggered by positive feed-back effect of low serum thyroxine, described as 'specificity spill over', ${ }^{, 23}$. But, the serum gonadotropins levels in these patients are relatively low in contrast to the degree of gonadal stimulation. Thus, elevated gonadotropins alone cannot completely explain the gonadal stimulation seen in severe juvenile hypothyroidism. Whereas, TSH level remain consistently elevated in such patients and the tendency to manifest sexual precocity may be directly related to the severity of $\mathrm{TSH}_{\text {elevation }}{ }^{2}$. It is also proposed that prolactin may play a primary role in the disease process, perhaps by sensitizing the ovaries to gonadotropins or that TSH itself sensitizes the ovaries to gonadotropin stimulation $^{4}$. Besides, the majority of human hormones act through 7-transmembrane G proteincoupled receptors (GPCRs), all of which share common intracellular signalling pathways but little is known about their distribution, activity and cross reactivity in hormone excess conditions. TRHinduced TSH excess may be the common stimulator of the FSH receptor and possibly other GPCRs ${ }^{3}$. So, high circulating level of TSH act directly on FSH receptors may be the actual mediator of precocity ${ }^{1,5}$.

Using recombinant tools, it has been shown that human TSH can interact with the human FSH receptor to stimulate the adenylyl cyclase activity, without simultaneous stimulation of $\mathrm{LH}$ receptors explaining the low prepubertal $\mathrm{LH}^{1,5}$. So, relatively low FSH-like activity of $\mathrm{TSH}$ can be clinically significant at very high concentrations of $\mathrm{TSH}$, present in severe primary hypothyroidism. Hyperprolactinemia, another common finding, also present in our patient, has two etiologies. Some postulate that the thyrotroph hyperplasia in the pituitary compresses the pituitary stalk, thereby disrupting hypothalamic inhibition of prolactin. Besides, TRH is also known to stimulate prolactin. So, when thyroid hormone is low, TRH increases and can lead to high prolactin ${ }^{6}$. Again $\mathrm{TRH}$ induced hyperprolactinaemia likely to suppress the pituitary gonadotrophic axis particularly $\mathrm{LH}_{\text {level }}{ }^{3}$. The increased FSH or FSH like activity of $\mathrm{TSH}$ and low $\mathrm{LH}$ cause a high $\mathrm{FSH} / \mathrm{LH}$ ratio in contrast to high LH/FSH ratio in normal puberty, thereby causing the increased ovarian estrogen secretion and multicystic ovaries $^{2}$. In females, the multicystic ovaries may result from elevated levels of circulating gonadotropins acting on it. However, ovarian enlargement may be secondary to a myxedematous infiltration ${ }^{1}$. A direct effect of severe hypothyroidism on the pre-pubertal testis leads to over proliferation of Sertoli cells is responsible for testicular enlargement in boys ${ }^{1,5}$. Another unique feature of VWGS is short stature and delayed bone age which differs from other causes of precocious puberty where growth acceleration is the norm. This can be explained on the basis that thyroid hormone mediated bone maturation involves both direct and indirect actions. The indirect action is mediated by the regulation of growth hormone gene expression and the insulin-like growth factor (IGF) system, while T3 directly regulates the endochondral ossification and also controls chondrocyte differentiation in the growth plate both in vitro and in vivo ${ }^{5}$. Summary of findings in Van Wyk and Grumbach syndrome ${ }^{1-6}$ :

Physical characteristics:

- Hypothyroid facies

-Short stature

-Precocious uterine bleeding

-Precocious thelarche

$\bullet \pm$ Galactorrhoea

-Absence of pubic hair or axillary hair.

Radiological characteristics:

-Enlarged and multicystic ovaries

-Pubertal uterus.

-Delayed bone age

-Enlarged pituitary

Endocrine abnormalities:

-Extremely raised TSH and low undetectable free T4

- High/ high normal FSH

-Suppressed LH

-Raised Prolactin

-Flat response on LHRH stimulation test

\section{Conclusion}

The precocious puberty in VWGS is unique in that it is classically associated with delayed bone age and short stature with severe hypothyroidism. However, the bone age is advanced in all cases of precocious puberty other than VWGS. The delayed bone age in our case along with high TSH, low T3, low T4, low 
LH levels and precocious puberty helped us to think of VWGS. In the absence of suspected ovarian torsion, surgery is unnecessary, as ovarian cyst regression occurs and all the hormones return to normal after appropriate thyroid hormone replacement.

\section{References}

1. Rastogi A, Bhadada SK, and Bhansali A. An unusual presentation of a usual disorder: Van WykGrumbach syndrome. Indian J Endocrinol Metab 2011 July; 15: 141-3.

2. Sneha LM, Thanasegarapandian K, Paramasivam $V$ et al. Short stature and an interesting association: Indian J Hum Genet 2013;19(1):101-3.

3. Baranowski E and Hogler W. Unusual presentations of a girl with Down syndrome: Van Wyk-Grumbach syndrome. European Journal of Endocrinology 2012; 166: 537-42.
4. Ryan GL, Feng X, d'Alva CB et al. Evaluating the Roles of Follicle-Stimulating Hormone Receptor Polymorphisms in Gonadal Hyperstimulation Associated with Severe Juvenile Primary Hypothyroidism 2007; 92(6):2312-7. available at http://press.endocrine.org/doi/pdf/10.1210/jc.2006-2 086.

5. Omran A, Peng J, Shrestha Bet al. Male Child with Van Wyk-Grumbach's Syndrome and Other Complications of Long-Standing Primary Hypothyroidism-A Case Report. Case Reports in Pediatrics 2012; Article ID 352751, 5 pages.

6. Durbin KL, Diaz-Montes T, Loveless MB. Van wyk and grumbach syndrome: an unusual case and review of the literature. J Pediatr Adolesc Gynecol 2011 Aug; 24(4): 93-6. 